\title{
ATTRACTORS: PERSISTENCE, AND DENSITY OF THEIR BASINS ${ }^{1}$
}

BY

\author{
MIKE HURLEY
}

\begin{abstract}
An investigation of qualitative features of flows on manifolds, in terms of their attractors and quasi-attractors. A quasi-attractor is any nonempty intersection of attractors. It is shown that quasi-attractors other than attractors occur for a large set of flows. It is also shown that for a generic flow (for each flow in a residual subset of the set of all flows), each attractor "persists" as an attractor of all nearby flows. Similar statements are shown to hold with "quasi-attractor", "chain transitive attractor", and "chain transitive quasi-attractor" in place of "attractor". Finally, the set of flows under which almost all points tend asymptotically to a chain transitive quasi-attractor is characterized in terms of stable sets of invariant sets.
\end{abstract}

0. Introduction. This paper investigates qualitative properties of attractors of flows on compact manifolds. An attractor of a flow, $f$, is a nonempty, compact, $f$-invariant set $A$ that has a neighborhood, $U$, satisfying $\cap_{t \geqslant 0} f(t, U)=A$. The basin of attraction of the attractor $A$ is the set of all points in the manifold that approach $A$ asymptotically under the forward flow.

The central concern is to determine when there is a collection of attractors of a given flow such that:

(a) Each attractor in the collection is "indecomposable" in some sense.

(b) Each of these attractors is "persistent" under perturbations of the flow.

(c) The union of the basins of these attractors forms a dense subset in the manifold.

The origin of the problem is a conjecture by $\mathrm{R}$. Thom:

CONJECTURE [T, p. 39]. Given $r \geqslant 1$, there is a set of flows, $S$, residual in the set of all $C^{r}$ flows on $M$, such that $f \in S$ implies that $f$ has a finite number of structurally stable, topologically transitive attractors whose basins are dense in $M$.

Note that the attractors Thom is talking about satisfy strong forms of the notions of "indecomposability" (topological transitivity) and "persistence" (structural stability). The primary examples of the exact situation Thom describes are the Axiom $A$ and no cycles flows (see [S] or [N] for definitions), except of course that these flows do not in general form a residual subset. In the time since the original conjecture was made, a number of examples have shown that the conjecture needs

Received by the editors October 6, 1980. Portions of the paper were presented in the Special Session on Differentiable Dynamical Systems at the A.M.S. Annual Meeting in San Antonio, January 1980.

1980 Mathematics Subject Classification. Primary 58F12; Secondary 54H20, 58F10.

Key words and phrases. Attractor, quasi-attractor, chain recurrence, chain transitivity.

'This paper describes work done in the author's thesis at Northwestern University. 
to be extensively modified if there is to be any chance of proving the residuality condition.

The first of these examples are due to S. Newhouse [Ne1], [Ne2]. They show that if the dimension of $M$ is greater than or equal to three and $r \geqslant 2$, then there is a second category set of $C^{r}$ flows on $M$, each one of which has an infinite number of hyperbolic periodic sinks.

A second important example was provided by M. Shub [HPS, §8], who showed that there is an open set of $C^{r}$ flows on the five-torus, $r \geq 1$, each one of which has the whole five-torus as its nonwandering set (and consequently as its only attractor), but none of which is structurally stable.

Finally, the work of J. Guckenheimer and Bob Williams on the Lorenz attractors [G, Wi2] shows that these attractors are not even locally of the same homeomorphism type (that is, not "topologically stable").

These examples show that both the finiteness and the stability aspects of Thom's original conjecture are too strong. Thus, in trying to reformulate the conjecture we are forced to choose a rather weak notion of "permanence". In addition, we choose a weaker version of "indecomposability" than topological transitivity. Our notion of permanence is called persistence. We say an attractor $A$ of $f$ is persistent if, whenever $f_{n}$ is a sequence of flows approaching $f$, then each $f_{n}$ has an attractor $A_{n}$ such that $A_{n}$ approaches $A$ in the Hausdorff topology. This is a weak property, but a generic one.

As mentioned above, we also have chosen a weakened version of indecomposability. In doing so we have taken advantage of a natural connection between the set of attractors of a flow and the chain recurrent set of that flow. This connection is made precise in the following theorem of C. Conley [C].

TheOREM (CONLEy). $C R(f)=\bigcap\{A \cup(M-B(A)) \mid A$ is an attractor of $f\}$. (Here $C R(f)$ is the chain recurrent set of $f$, and $B(A)$ is the basin of attraction of the attractor $A$.)

In particular, the theorem tells us that if the set of attractors of $f$ is ordered by inclusion, then any minimal elements in this ordering will be chain recurrent. Hence we take chain recurrence to be our notion of indecomposability. The problem is that the set of attractors of a flow is not closed under arbitrary intersections, so there may be no minimal elements in this ordering by inclusion. The remedy for this problem is just to include the sets formed by these intersections in the class of sets under consideration. Hence we introduce the notion of quasi-attractor. $X$ is a quasi-attractor if $X$ is nonempty and can be realized as $\cap_{n} A_{n}$ where each $A_{n}$ is an attractor. (Note that under this definition every attractor is a quasi-attractor.) If the set of all quasi-attractors of a flow $f$ is ordered by inclusion, then minimal elements will always exist and will be chain recurrent.

Now we can reformulate Thom's conjecture as follows:

Conjecture 1. For a residual subset of the set of $C^{r}$ flows on $M, D(f)$ is dense in $M$, where $D(f)$ is the union of the basins of the chain recurrent quasi-attractors. 
Some progress toward an affirmative answer to this conjecture is made in this paper. In particular,

TheOREM A (7.21). There is a residual subset, $I_{r}$, of the set of $C^{r}$ flows, for any $r \geqslant 0$, such that if $f \in I_{r}$, then:

(a) Every attractor of $f$ is persistent.

(b) Every quasi-attractor of $f$ is persistent.

(c) Every chain recurrent attractor of $f$ persists as a chain recurrent attractor.

(d) Every chain recurrent quasi-attractor of $f$ persists as a chain recurrent quasiattractor.

In terms of the density question we have defined an apparently weak property of flows, called the generalized saddle property, and shown that it is equivalent to the density of $D(f)$. This property requires that whenever $X$ is an $f$-invariant set whose stable set has interior, then $X$ contains a quasi-attractor.

THEOREM B (6.5). The following are equivalent:

(a) $f$ satisfies the generalized saddle property.

(b) The basins of the chain transitive quasi-attractors of $f$ form a dense subset of $M$.

(c) The basins of the chain transitive quasi-attractors of $f$ form a residual subset of M.

CONJECTURE 2. The generalized saddle property is generic.

Note that Conjectures 1 and 2 are equivalent in light of Theorems $\mathbf{A}$ and $\mathbf{B}$. While we have been unable to either prove or disprove these conjectures in the general case, the $C^{0}$ case follows as a corollary of Shub's density theorem [Sh1].

THEOREM C (6.12). There is a residual subset of the set of $C^{0}$ flows on $M$, each flow of which has the union of the basins of its chain transitive quasi-attractors forming a residual subset of $M$.

In proving these results we introduce an analogue of the omega limit set, called the chain-omega limit set, denoted $Z(x)$. It is defined to be the maximal chain transitive set containing the omega limit set of $x$; the exact definition is in 4.1. Like the omega limit set, $Z(x)$ is closed and invariant. It is a natural object to consider when working with chain transitive quasi-attractors because $x$ is in the basin of a chain transitive quasi-attractor if and only if $Z(x)$ is a quasi-attractor.

The major influences on this paper are Conley's work on chain recurrence and attractors, and Takens' work on the tolerance stability conjecture. I should also mention that it is purely for expository reasons that the results are stated in terms of flows, as opposed to vector fields or diffeomorphisms, and that all of the main results hold true in these other contexts.

The first three sections of this paper establish the basic facts and definitions. $\$ 4$ presents the sets $Z(x)$ described above. $\$ 5$ contains preliminaries to Theorems $\mathrm{A}, \mathrm{B}$, and C. In $\$ 6$ Theorems $B$ and $C$ are proven, while Theorem A is proven in $\$ 7$.

I thank my advisor, Clark Robinson, for his patient help over the past few years. Thanks also to John Franks, Bob Williams, and Lai-Sang Young for their help, and to Bob Devaney for starting it all. 


\section{Definitions and basic facts.}

A. Notation. Suppose $X$ is a topological space, $Y$ is a subset of $X, x$ is a point in $X$, and $f$ is a flow on $X$ ("flow" is defined in (vi) below).

(i) $\operatorname{clos}(Y)$ is the closure of $Y$ in $X$.

(ii) $\operatorname{int}(Y)$ is the interior of $Y$ in $X$.

(iii) $\partial Y$ is the topological boundary of $Y$ in $X$, that is, $\partial Y=\operatorname{clos}(Y)-\operatorname{int}(Y)$.

(iv) If $d$ is a metric on $X$, then $U_{\varepsilon}(x)=\{y \in X \mid d(x, y)<\varepsilon\}$.

(v) $R, R^{+}$, and $R^{-}$stand for $(-\infty, \infty),[0, \infty)$ and $(-\infty, 0]$, respectively.

(vi) A flow $f$ on $X$ is a continuous (or smooth) map $f: R \times M \rightarrow M$ that satisfies the group property in the first variable; that is, $f(0, x)=x$, and $f(s+t, x)=$ $f(s, f(t, x))$ for all $x$ in $M$ and all $s$ and $t$ in $R$.

(vii) $O(x), O^{+}(x)$, and $O^{-}(x)$ respectively denote the orbit, forward orbit, and backward orbit of $x$ under $f$. In other words:

$$
\begin{aligned}
& O(x)=\{f(t, x) \mid t \in R\} . \\
& O^{+}(x)=\left\{f(t, x) \mid t \in R^{+}\right\} \\
& O^{-}(x)=\left\{f(t, x) \mid t \in R^{-}\right\}
\end{aligned}
$$

(viii) Given $f$ and a point $x$ in $X$, we can form the omega limit set of $x$, $\omega(x)=\bigcap_{t \geqslant 0} \operatorname{clos}(f([t, \infty), x))$. If $X$ is compact, $\omega(x)$ is always nonempty. The alpha limit set of $x$ is defined analogously; $\alpha(x)=\bigcap_{t<0} \operatorname{clos}(f(-\infty, t], x)$.

(ix) A point $x$ in $X$ is called wandering for $f$ if there is a neighborhood $N$ of $x$ and a time $t \geqslant 0$ such that $f([t, \infty), N) \cap N=\varnothing$. Any point that is not wandering is called nonwandering. The set of all nonwandering points of $f$ will be denoted $\Omega(f)$. This set is closed, $f$-invariant, and contains every alpha and omega limit set.

B. Attractors. The central notion in this work is that of attractor. Our definition is that used by Conley; that is, an attractor for $f$ is a compact, nonempty, $f$-invariant subset $A$ of $M$, such that $A$ has an open neighborhood $U$ satisfying $\cap_{t>0} f(t, U)=A$. The basin of attraction of $A$, denoted $B(A)$, is the set of all points in $M$ that approach $A$ asymptotically under the forward flow; that is, $B(A)=\{x \in M \mid \omega(x) \subset A\}$. If the neighborhood $U$ of $A$ described above is chosen carefully, then $B(A)=\cup_{t \leqslant 0} f(t, U)$. (How to make this choice of $U$ is described in 1.6.) $B(A)$ is open, $f$-invariant, and a neighborhood of $A$. Note that we make no special assumptions about the action of the flow on the attractor; in particular, the whole manifold is always an attractor for any flow on it.

Remarks. 1.1 If $A, A^{\prime}$ are attractors with $A \cap A^{\prime} \neq \varnothing$, then $A \cap A^{\prime}$ is an attractor as well.

1.2 If $A$ is an attractor and $A=A_{1} \cup A_{2}$, with each $A_{i}$ compact and $A_{1} \cap A_{2}=$ $\varnothing$, then each $A_{i}$ is an attractor.

1.3 If $A$ is an attractor and $\alpha(x) \cap A \neq \varnothing$, then $x \in A$.

1.4 The number of attractors of $f$ is countable.

1.5 If $\gamma$ is a hyperbolic periodic orbit in an attractor $A$, then the unstable manifold of $\gamma$ is contained in $A$. See [N] or [S] for definitions.

The proofs of these remarks are easy and are omitted here; a general reference is [C].

The following lemma shows that we can find neighborhoods of an attractor that are "well behaved" under the flow. 
1.6 LEMMA. If $A$ is an attractor and $\varepsilon>0$, then there is an open set $V$ satisfying:

(i) $A \subset V \subset U_{\varepsilon}(A)$.

(ii) $\cap_{t>0} f(t, \operatorname{clos}(V))=A$.

(iii) $f(t, V) \subset V$ for all $t \geqslant 0$.

(iv) If $G$ is any neighborhood of $A$, then there is a constant $n(G) \geqslant 0$ such that $t>n(G)$ implies that $f(t, \operatorname{clos}(V)) \subset G$.

Proof. Let $U_{0}$ be a defining open neighborhood of $A$ and let $U$ be open in $M$ and satisfy $A \subset U \subset \operatorname{clos}(U) \subset U_{0}$. Define $V=\left\{x \mid O^{+}(x) \subset U\right\}$. Clearly $A \subset V$ $\subset U$, and $V$ is mapped into itself under the forward flow of $f$, so (i)-(iii) are clear. (iv) follows directly from (ii) and (iii), since they imply that

$$
A=\bigcap_{t>0} f(t, \operatorname{clos}(V))=\lim _{t \rightarrow \infty} f(t, \operatorname{clos}(V)),
$$

where we take this last limit in the Hausdorff topology (see 1.C below for the definition of this topology). All that remains is to show that $V$ is open. We do this by proving that its complement is closed. Let $W=\left\{x \in \operatorname{clos}(U) \mid O^{+}(x) \cap M-U\right.$ $\neq \varnothing\}$. As $M-V=W \cup(M-U)$, it is enough to show that $W$ is closed. Suppose that $x_{n} \in W$, and $x_{n} \rightarrow x$ in $M$. Clearly $x \in \operatorname{clos}(U)$. By assumption, there are real numbers $t_{n} \geqslant 0$ with $f\left(t_{n}, x_{n}\right) \in M-U$. There are two cases, depending upon whether the sequence $\left\{t_{n}\right\}$ is bounded or not. If it is bounded, then by taking a subsequence we may assume that $t_{n} \rightarrow T \geqslant 0$. Then $f(T, x)=\lim f\left(t_{n}, x_{n}\right) \in M$ $-U$, and so $x \in W$ as required. The second case is when $\left\{t_{n}\right\}$ is unbounded. Without loss of generality we may assume that these $t_{n}$ were chosen so that if $s \in\left[0, t_{n}\right)$, then $f\left(s, x_{n}\right) \in U$. If we denote $f\left(t_{n}, x_{n}\right)$ by $y_{n}$, we now know that:

(a) Each $y_{n} \in \partial U$, and

(b) $f\left(-s, y_{n}\right) \in U$ for $s \in\left(0, t_{n}\right]$.

By taking subsequences we may also assume that:

(c) $\lim t_{n}=\infty$.

(d) $y_{n}$ converges to some point $y \in \partial U$.

Combining (b)-(d) we must conclude that $O^{-}(y) \subset \operatorname{clos}(U)$; that is, $f(-t, y) \in$ $\operatorname{clos}(U)$ for all $t \geqslant 0$, so $y \in f(t, \operatorname{clos}(U))$ for all $t \geqslant 0$. Then $y \in$ $\cap_{t>0} f(t, \operatorname{clos}(U))=A$. However, our construction ensures that $y \in \partial U$; since $A \subset \operatorname{int}(U)$ this is a contradiction, so the second case can never occur. Hence we conclude that $W$ is closed, and so $V$ is open.

We finish this subsection with the following definition:

1.7 Definition. Given a flow $f$, define a new flow $f^{*}$ by $f^{*}(t, x)=f(-t, x)$. $f^{*}$ has the same orbits as $f$, but the direction of the flow lines is reversed. We say $X$ is a repellor for $f$ if it is an attractor for $f^{*}$; its basin of repulsion is denoted $B^{*}(X)$ and is defined to be the basin of attraction of $X$ with respect to $f^{*}$. Note that if $A$ is an attractor for $f$, then $M-B(A)$ is a repellor and $B^{*}(M-B(A))=M-A$. For a more detailed description, see [C].

C. The Hausdorff topology. Given a compact metric space $(X, d)$, consider the set $F X$ composed of all the nonempty closed subsets of $X$. Given two sets $Y$ and $Y^{\prime}$ in $F X$, let $d_{H}\left(Y, Y^{\prime}\right)=\inf \left\{\alpha>0 \mid Y \subset U_{\alpha}\left(Y^{\prime}\right)\right.$ and $\left.Y^{\prime} \subset U_{\alpha}(Y)\right\}$, where, as usual, $U_{\alpha}\left(\_\right)$denotes the $\alpha$-ball measured in $d_{\text {. }} d_{H}$ is a metric on $F X$, called the Hausdorff 
metric. The topology it induces makes $F X$ a compact space. For proofs of these facts, see [Na, Chapter 0] or [K, §17].

Note that we may now add the empty set to $F X$ just by making it an isolated point; we can extend the definition of $d_{H}$ to this larger setting by defining $d_{H}(\varnothing, \varnothing)=0$, and for $Y \neq \varnothing, d_{H}(Y, \varnothing)=k$, where

$$
k>\sup \left\{d_{H}\left(Z, Z^{\prime}\right) \mid Z, Z^{\prime} \in F X-\{\varnothing\}\right\} .
$$

In the sequel we assume this to be the definition of $F X$.

Two final remarks are in order. First, one can extend the definition of $d_{H}$ to all subsets of $M$, although it is only a semimetric on this set, as, for example, $d_{H}(X, \operatorname{clos}(X))=0$ for all $X \subset M$. Secondly, once this extension has been made, it is not difficult to see that for any subsets $X, Y$ of $M$,

$$
d_{H}(X, Y)=d_{H}(M-X, M-Y) .
$$

D. Genericity. Recall that a subset of a complete metric space is called residual if it can be realized as the intersection of a countable number of dense open sets. By the Baire category theorem any residual subset of a complete metric space is dense in that space. A property of flows is said to be $C^{r}$ generic $(r>0)$ if it is true of a residual subset of $F^{r}(M)$, the set of $C^{r}$ flows on $M$ with the $C^{r}$ compact-open topology. (That is, two flows are close if they and their derivatives up to order $r$ are uniformly close on $[-1,1] \times M$.) With this topology $F^{r}(M)$ is a complete metric space [Hi].

We denote the $C^{r}$ metric on $F^{r}(M)$ by $d_{r}$.

One way of describing residual sets is through the use of semicontinuous functions.

1.8 Definition. Suppose $\left(X_{i}, d_{i}\right)$ are metric spaces, $i=1$ or 2 , and that $X_{2}$ is compact. Let $\left(F X_{2}, d_{H}\right)$ be the set of closed subsets of $X_{2}$ equipped with the Hausdorff metric. A map $f: X_{1} \rightarrow F X_{2}$ is said to be upper semicontinuous at $x \in X_{1}$ if, given any number $\alpha>0$, there exists $\beta=\beta(\alpha, x)>0$ such that $f(y) \in$ $U_{\alpha}(f(x))$ whenever $d_{1}(x, y)<\beta$. The map $f$ is lower semicontinuous at $x$ if, given $\alpha>0$, there is a $\beta>0$ such that $f(x) \subset U_{\alpha}(f(y))$ whenever $d_{1}(x, y)<\beta$. The map $f$ is said to be upper (lower) semicontinuous on $X_{1}$ if it is upper (lower) semicontinuous at $x$ for all $x$ in $X_{1}$. See [Na, Chapter 0$]$ and [K, \$18].

The importance of these notions for the study of generic properties is contained in the following theorems.

1.9 THEOREM. If $X$ and $Y$ are metric spaces with $Y$ compact, and if $f: X \rightarrow F Y$ is either upper or lower semicontinuous, then the set of continuity points of $f$ is a residual subset of $X$.

Proof. See [Ta1, Lemma 2.3].

1.10 TheOREM. Suppose that $X$ and $Y$ are metric spaces with $Y$ compact, and that $g: X \times Y \rightarrow R$ is continuous. Define $\bar{g}: X \rightarrow F Y$ by $\bar{g}(x)=\{y \in Y \mid g(x, y)=0\}$. Then $\bar{g}$ is upper semicontinuous. 
Proof. See [Ta1, Lemma 2.4].

1.11 REMARK. Suppose $X$ is a metric space and $f$ is a map from $X$ into $R^{+}$. Let $Y$ be the one-point compactification of $R^{+}$, and let $\bar{f}$ be the map from $X$ into $F Y$ defined by $\bar{f}(x)=[0, f(x)]$. We say $f$ is upper (lower) semicontinuous if $\bar{f}$ is. Note that this agrees with the usual definition of semicontinuity for real-valued functions, that is, $f$ is upper semicontinuous at $x$ if, whenever $x_{n} \rightarrow x$, then $\lim \sup f\left(x_{n}\right) \leqslant f(x)$, and $f$ is lower semicontinuous at $x$ if whenever $x_{n} \rightarrow x$, $\lim \inf f\left(x_{n}\right) \geqslant f(x)$. We define lower semicontinuity for functions into the extended half-line, $[0, \infty]$, in the same way.

\section{Chain recurrence.}

2.1 Definition. An $(\varepsilon, t)$-chain for $f$ is a sequence of orbit segments, $\left\{G_{n}\right\}$, $n=0,1, \ldots, p$, each one of the form $G_{n}=f\left(\left[0, t_{n}\right), x_{n}\right)$ and satisfying:

(i) $t_{n} \geqslant t$ for every $n$.

(ii) $d\left(f\left(t_{n}, x_{n}\right), x_{n+1}\right)<\varepsilon$.

We say the chain goes through $y$ if $y \in G_{n}$ for some $n$, we say the chain goes to $y$ if $y=x_{n}$ for some $n \geqslant 1$, and we say it comes from $x_{0}$. Sometimes we omit explicit reference to the $t_{i}$ 's and denote the chain as $x_{0} \rightarrow x_{1} \rightarrow \cdots \rightarrow x_{p}$.

2.2 Definition. The chain recurrent set for $f$, denoted $C R(f)$, is the set $\{x \mid$ for each $\varepsilon>0$ and $t \geqslant 0$ there is an $(\varepsilon, t)$-chain from $x$ to $x\}$. The definition of $C R(f)$ is due to Conley. The notion of $(\varepsilon, t)$-chains has also been studied by many others, including Anosov, Bowen, and Sinai. See p. 88 of [B1] for a short discussion of the history.

2.3 REMARK. $C R(f)$ is closed, invariant, and contains the nonwandering set [C].

2.4 LEMMA. In the definition of $C R(f)$ it suffices to fix $t=1$.

Proof. Define $h: R^{+} \rightarrow R^{+}$to be a strictly positive (but not necessarily continuous) function that satisfies: $d(x, y)<h(\varepsilon)$ and $0 \leqslant t \leqslant 2$ imply that $d(f(t, x), f(t, y))<\frac{1}{2} \varepsilon$. We claim that:

(a) $h(\varepsilon)<\frac{1}{2} \varepsilon$.

(b) For any $p \geqslant 1$ and $1 \leqslant j \leqslant p, h^{p}+\frac{1}{2} h^{p-j}<h^{p-j} .\left(h^{p}(\varepsilon)=h\left(h^{p-1}(\varepsilon)\right)\right.$; $\left.h^{0}(\varepsilon)=\varepsilon\right)$.

(c) If $\left\{\left(x_{i}, t_{i}\right) \mid i=1,2, \ldots, p\right\}$ is an $\left(h^{p}(\varepsilon), 1\right)$-chain with each $t_{i} \leqslant 2$, then

$$
d\left(f\left(t_{p}, x_{p}\right), f\left(\sum_{i=1}^{p} t_{i}, x_{1}\right)\right)<\frac{\varepsilon}{2} .
$$

The proofs of these claims are as follows:

(a) is clear from the definitions. The proof of $(b)$ is by induction on $j$. For any $p \geqslant 1$ and $j=1$,

$$
h^{p}+\frac{1}{2} h^{p-1}=h\left(h^{p-1}\right)+\frac{1}{2} h^{p-1}<2\left(\frac{1}{2} h^{p-1}\right)=h^{p-1} \text { by }(\mathrm{a}) .
$$

Now suppose that we know the result for a given value of $j$ and for all $p \geqslant j$; we wish to then prove it for $j+1$ and all $p \geqslant j+1$. If $p=1$, there is nothing to 
prove; for $p>1$, we have

$$
\begin{aligned}
h^{p}+\frac{1}{2} h^{p-(j+1)} & =h^{p}+\frac{1}{2} h^{(p-1)-j}<h^{p-1}+\frac{1}{2} h^{(p-1)-j} \\
& <h^{(p-1)-j}=h^{p-(j+1)}
\end{aligned}
$$

where we have used the induction hypothesis to get the last inequality.

The proof of (c) is by induction on $p$. If $p=1$, there is nothing to prove. Suppose the result is known for a given $p$; we shall establish it for $p+1$. Let $\left\{\left(x_{i}, t_{i}\right) \mid\right.$ $i=1,2, \ldots, p+1\}$ be an $\left(h^{p+1}(\varepsilon), 1\right)$-chain; as $h^{p+1}(\varepsilon)=h^{p}(h(\varepsilon))$ we see that $\left\{\left(x_{i}, t_{i}\right) \mid i=1,2, \ldots, p\right\}$ is an $\left(h^{p}(h(\varepsilon)), 1\right)$-chain, so by the induction hypothesis we have

(d) $d\left(f\left(t_{p}, x_{p}\right), f\left(\sum_{i=1}^{p} t_{i}, x_{1}\right)\right)<\frac{1}{2} h(\varepsilon)$.

Also, by definition of the chain,

(e) $d\left(f\left(t_{p}, x_{p}\right), x_{p+1}\right)<h^{p}(\varepsilon)$.

Adding (d) and (e), we see that

$$
d\left(x_{p+1}, f\left(\sum_{i=1}^{p} t_{i}, x_{1}\right)\right)<h^{p}(\varepsilon)+\frac{1}{2} h(\varepsilon) .
$$

If $p>1$, then (b) applies to show that this last expression is less than $h(\varepsilon)$. If $p=1$, replace inequality (d) by equality $\left(\mathrm{d}^{\prime}\right): d\left(f\left(t_{1}, x_{1}\right), f\left(t_{1}, x_{1}\right)\right)=0$, and we still get $d\left(x_{2}, f\left(t_{1}, x_{1}\right)\right)<h^{p}(\varepsilon)=h(\varepsilon)$. In either case, we now use the definition of $h(\varepsilon)$ to conclude that $d\left(f\left(t_{p+1}, x_{p+1}\right), f\left(\sum_{i=1}^{p+1} t_{i}, x_{1}\right)\right)<\frac{1}{2} \varepsilon$.

Now we prove the lemma. Let $x$ be a point such that for every $\delta>0$ there is a $(\delta, 1)$-chain from $x$ to $x$, and let $\varepsilon$ and $s>0$ be given. We shall construct an $(\varepsilon, s)$-chain from $x$ to itself. Let $p$ be the greatest integer in $s+1$ and let $\delta=h^{p}(\varepsilon)$. Let $\left\{\left(x_{i}, t_{i}\right) \mid i=1,2, \ldots, k p+r\right\}$ be an $\left(h^{P}(\varepsilon), 1\right)$-chain with $k \geqslant 0,0 \leqslant r<p$ and each $t_{i} \leqslant 2$. (We can always get such a chain with arbitrary $t_{i}$; from this original chain we can get one of the desired type merely by partitioning any longer interval $\left[0, t_{i}\right)$ into pieces of the desired length.) By concatenating this chain $p$ times we can assume $r=0$. Now by (c), $d\left(f\left(\sum_{i=1}^{p} t_{i}, x_{i}\right), f\left(t_{p}, x_{p}\right)\right)<\frac{1}{2} \varepsilon ;$ also, $d\left(f\left(t_{p}, x_{p}\right), x_{p+1}\right)<$ $h^{p}(\varepsilon)<\frac{1}{2} \varepsilon$. Thus $\left(x, \sum_{i=1}^{p} t_{i}\right) \rightarrow x_{p+1}$ is an $(\varepsilon, s)$-chain $\left(\sum_{i=1}^{p} t_{i} \geqslant p \geqslant s\right)$. Similarly, for any $j=0,1,2, \ldots k,\left(x_{j p+1}, \sum_{i=j p+1}^{(j+1) p} t_{i}\right) \rightarrow x_{(j+1) p+1}$ is an $(\varepsilon, s)$-chain, and thus $x=x_{1} \rightarrow x_{p+1} \rightarrow x_{2 p+1} \rightarrow \cdots \rightarrow x_{k p+1}=x$ is an $(\varepsilon, s)$-chain from $x$ to $x$. Hence $x$ is chain recurrent and the lemma is proven.

2.5 Definition. A nonempty set $V$ is said to be $(\varepsilon, 1)$-chain transitive if for all $x, y$ in $V$ there are $(\varepsilon, 1)$-chains from $x$ through $y$ and from $y$ through $x$. We say $V$ is chain transitive if it is $(\varepsilon, 1)$-chain transitive for all $\varepsilon>0$.

2.6 Definition. A maximal chain transitive set is called a chain component. (Here maximal means maximal by inclusion.) Note that chain components are compact and invariant. Similarly, for $\varepsilon>0$, a maximal $(\varepsilon, 1)$-chain transitive set is called an $(\varepsilon, 1)$-chain component.

2.7 REMARK. If $X$ is connected and chain recurrent, then $X$ is chain transitive.

The reason for our interest in chain recurrence is that it is a natural notion of "indecomposability" for attractors. The reason for this is the following theorem of C. Conley. 
2.8 TheOREM (CONLEY). $C R(f)=\cap\{A \cup(M-B(A)) \mid A$ is an attractor for $f\}$.

Proof. [C, p. 37].

2.9 REMARK. In particular, Conley's theorem tells us the following: If we define a partial ordering on the set of attractors of $f$ by $A \leqslant A^{\prime}$ if and only if $A \subseteq A^{\prime}$, then minimal elements in this ordering will be chain components of $f$.

Proof. Suppose $A$ is minimal and let $x \in A$. If $x$ is not chain recurrent, then the theorem asserts the existence of an attractor $A^{\prime}$ such that $x \in B\left(A^{\prime}\right)-A^{\prime}$. Thus $\omega(x) \subset A^{\prime}$. As $A$ is closed, invariant and contains $x, \omega(x) \subset A$ as well. Thus $A \cap A^{\prime} \neq \varnothing$; by 1.1 and rininimality, we have $A \subset A^{\prime}$ and so contradict the assumption that $x \notin A^{\prime}$. This shows that $A \subset C R(f)$. Using 1.2 it is easy to see that minimality implies that $A$ is connected. Finally, 2.7 applies to show that $A$ is chain transitive.

2.10 REMARK. These results give a description of the attractors that are minimal in this ordering. However, there may be no minimal elements in this ordering for a given flow. The remedy for this problem is to extend the class of sets under consideration; this extension will be discussed in the next section.

\section{Quasi-attractors.}

3.1 Definition. A quasi-attractor for $f$ is a compact, nonempty, $f$-invariant set $X$ of the form $X=\cap_{n} A_{n}$, with each $A_{n}$ an attractor of $f$.

Note that under this definition, "quasi-attractor" is a more general notion than "attractor". That is, every attractor is a quasi-attractor. The converse of this statement is false, as the following simple example shows.

3.2 Example. View $S^{1}$ as the one-point compactification of $R^{1}$. Put a source at infinity, and for each $n=0,1,2 \ldots$, a sink at $1 /(2 n+1)$, and a source at $1 /(2 n+2)$. The origin is a quasi-attractor but not an attractor.

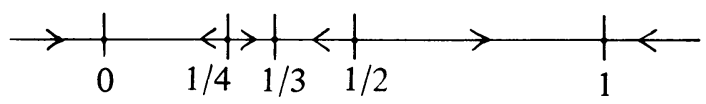

FIGURE 3.2

3.3 REMARK. If we order the set of all quasi-attractors of $f$ by inclusion (see 2.10), then minimal elements will exist and will be chain transitive. That is, every quasi-attractor contains a chain transitive quasi-attractor.

ProOF. Since any nonempty intersection of quasi-attractors is a quasi-attractor, Zorn's Lemma applies to show the existence of the minimal elements. The proof that they are chain transitive follows the same lines as the proof in 2.9.

In the remainder of this section we describe some of the differences between attractors and quasi-attractors.

3.4 REMARK. The number of quasi-attractors of $f$ is not necessarily countable (compare with 1.4). For example, let $R_{1}$ be a square in the plane and suppose the flow takes it into its interior in forward time. Inside $R_{1}$ there are two more squares, $R_{12}$ and $R_{11}$, and a saddle not contained in either, as indicated in the diagram below. 


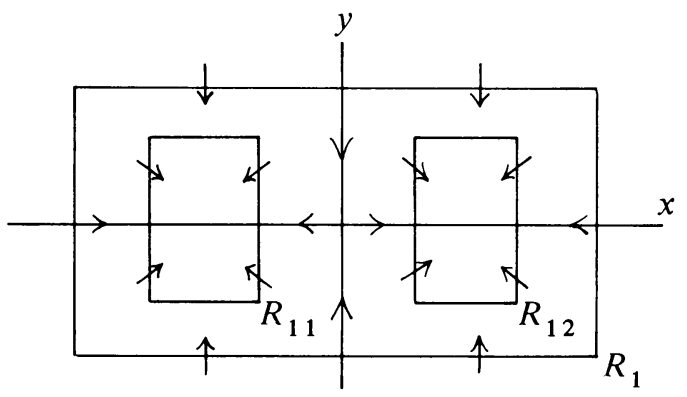

F IGURE 3.3

As indicated in the diagram, we assume that each of the smaller squares is also mapped into its interior; we also assume that the flow on the interior of each of these squares is exactly the same as the flow on $R_{1}$. At the $n$th stage of this construction, we will have $2^{n}$ squares, each mapped into itself. The intersection of all the forward iterates of a given square will be an attractor; taking intersections of nested sequences of these attractors gives quasi-attractors that are not attractors. It is not hard to see that the set of all these quasi-attractors is a Cantor set lying on the horizontal axis, and that each point of this Cantor set is a fixed point of $f$ and a quasi-attractor. Note that this example is essentially one dimensional; we have defined it in the plane for ease of description.

3.5 REMARK. A quasi-attractor is an attractor if and only if it is isolated in the chain recurrent set in the following sense: There exists a neighborhood $U$ of the quasi-attractor $X$ such that $U \cap C R(f) \subset X$.

In particular, suppose we have a sequence of attractors, $\left\{A_{n}\right\}$, with $A_{n+1} \subset A_{n}$ for each $n$. Then $\cap A_{n}$ is an attractor if and only if there is a number $n^{\prime}$ such that $A_{n^{\prime}}=A_{n^{\prime}+1}=A_{n^{\prime}+2}=\ldots$.

Now we show that quasi-attractors occur quite frequently in dynamical systems. The first result in this direction is the following theorem, which is merely a translation of work of S. Newhouse into the present setting.

3.6 Theorem (Newhouse). If $\operatorname{dim} M \geqslant 3$ and $r \geqslant 2$, then there is a second category subset $S$ (that is, $S$ is a residual subset of an open set) of the set of $C^{r}$ flows on $M$, each flow of which has a quasi-attractor that is not an attractor.

Proof. Newhouse's work shows that there is a second category set $S$, each flow of which has an infinite number of periodic sources [Ne2]. For $f \in S$, list these sources $\left\{\gamma_{n}\right\}$. Let $B\left(\gamma_{n}\right)$ denote the basin of repulsion of $\gamma_{n}$. Let $A_{k}=M-$ $\cup_{n=1}^{k} B\left(\gamma_{n}\right)$, so each $A_{k}$ is an attractor, and $A_{k+1} \subsetneq A_{k}$ for each $k$. By Remark 3.5, $\cap A_{k}$ is a quasi-attractor but not an attractor.

In a later section, (5.8), we shall strengthen this theorem. We close this section with a definition.

3.7 Definition. If $X$ is a quasi-attractor, we define the basin of $X, B(X)=\{x \in$ $M \mid \omega(x) \subset X\}$. Note that in contrast to the basin of an attractor, the basin of a quasi-attractor may not be open. 
4. Other limit sets. In this section we define and describe sets that are analogues of the omega limit sets but defined in terms of the notions of chain recurrence.

4.1 Definition. For $x \in M$, let $Z(x)=\{y \mid$ for each $\varepsilon>0$ and each $z \in \omega(x)$, there is an $(\varepsilon, 1)$-chain from $z$ to $y$ and from $y$ to $z$. In other words, $Z(x)$ is the chain component of $R$ that contains $\omega(x)$. This set is related to what Conley calls the chain-limit set; see 5.5 for the definition of this set and a discussion of the differences between the two.

4.2 Remark. For each $x, Z(x)$ is nonempty, closed, $f$-invariant and chain transitive.

The proofs are immediate.

4.3 Defintion. An even larger version of the limit set is obtained from the definition of $Z(x)$ by requiring the existence of $(\varepsilon, 1)$-chains only for $\varepsilon$ larger than some predetermined constant $\varepsilon_{0}$. To be precise, define $Z\left(x, \varepsilon_{0}\right)=\{y \in M \mid$ whenever $z \in \omega(x)$, then there are $\left(\varepsilon_{0}, 1\right)$-chains from $y$ to $z$ and from $z$ to $y$ \}. In other words, $Z\left(x, \varepsilon_{0}\right)$ is the maximal $\left(\varepsilon_{0}, 1\right)$-chain transitive set containing $\omega(x)$.

4.4 REMARK. (i) For each $\varepsilon>0, Z(x, \varepsilon)$ is open and $Z(x) \subset Z(x, \varepsilon)$.

(ii) $\cap_{e>0} Z(x, \varepsilon)=Z(x)$.

(iii) For a given $\varepsilon>0$, there are only finitely many distinct $Z(x, \varepsilon)$ 's.

The properties outlined in 4.4 are the heart of the advantage gained by using the sets $Z(x, \varepsilon)$ as approximations of the sets $Z(x)$. In addition, because these limit sets contain the nonwandering set and obey a transitivity condition (two of the $Z(x, \varepsilon)$ 's for a given $\varepsilon$ are either disjoint or identical), one can establish the following.

4.5 TheOREM. Let $f$ be a continuous flow on a compact space $X$. Then there is a residual subset $J$ of $X$, such that at every point of $J$ the map $x \rightarrow Z(x)$ is upper semicontinuous. That is, given $\alpha>0$, there is $\beta>0$ such that $d(x, y)<\beta$ implies $Z(y) \subset U_{\alpha}(Z(x))$.

Since we will not use 4.5 in the remainder of this paper, we omit the proof.

5. Admissible sequences and filtrations. In the first part of this section we give an alternate characterization of chain transitive quasi-attractors; in the second part we discuss ways of finding attractors using the notion of filtration and Conley's related concept of Morse decomposition [C]. In the final part we give a characterization of the flows that have quasi-attractors that are not attractors.

A. Admissible sequences.

5.1 Definition. An admissible sequence for $f$ is a finite, ordered set of chain components, $X_{0}, X_{1}, X_{2}, \ldots, X_{k}$, with $k>1$, satisfying: for each $\varepsilon>0$ and $i=$ $0,1,2, \ldots, k-1$, there is an $(\varepsilon, 1)$-chain from $X_{i}$ to $X_{i+1}$, and $X_{i} \neq X_{i+1}$.

5.2 REMARK. All of the chain components $X_{i}$ in an admissible sequence are distinct.

Proof. It clearly suffices to show $X_{0} \neq X_{k}$; if $k=1$ this is by definition; if $k>1$ and $X_{0}=X_{k}$, then all of the $X_{i}$ would have to be identical because of the maximality in the definition of chain component.

5.3 LEMMA. A chain component $X$ is a quasi-attractor if and only if no admissible sequence begins with $X$. 
Proof. (i) Suppose $X$ is a quasi-attractor; let $Y=\{x \mid$ for each $\varepsilon>0$, there is an $(\varepsilon, 1)$-chain from $X$ through $x$ \}. We shall show $Y=X$. Let $X_{n}$ be a sequence of attractors decreasing to $X$, that is, $X_{n+1} \subset X_{n}$ and $\cap_{n} X_{n}=X$. Let $r>0$ be given. Choose $n_{0}$ such that $n>n_{0}$ implies that $X_{n} \subset U_{r}(X)$. As $X_{n}$ is an attractor, there is a constant $\varepsilon>0$ such that every $(\varepsilon, 1)$-chain starting in $X_{n}$ stays in $U_{r}\left(X_{n}\right)$. It follows that $X \subset Y \subset U_{2 r}\left(X_{n}\right)$; as $r$ was arbitrary, $Y=X$.

(ii) Assume that no admissible sequence begins with $X$. Let $Y_{k}=\{x \mid$ there is a $(1 / k, 1)$-chain from $X$ through $x\}$. Now note:

(a) As no admissible sequence begins with $X, \cap_{k} Y_{k}=X$.

(b) Each $Y_{k}$ is forward invariant. This is because of the use of chains "through" $x$ in the definition of $Y_{k}$.

(c) For $t \geqslant 1, U_{1 / k}\left(f\left(t, Y_{k}\right)\right) \subset Y_{k}$. (If $t \geqslant 1$, then anything in the $(1 / k)$-ball about $f\left(t, Y_{k}\right)$ can be reached via a $(1 / k, 1)$-chain from $Y_{k}$ and hence from $X$.)

(d) By (b) and (c), if we define $A_{k}=\bigcap_{t \geqslant 0} f\left(t, Y_{k}\right)$, then each $A_{k}$ is an attractor and contains $X$, as $X$ is invariant.

(e) By (a) and (d), $X \subset \cap_{k} A_{k} \subset \cap_{k} Y_{k}=X$, so $X=\cap_{k} A_{k}$ is a quasiattractor.

B. Filtrations.

5.4 Definition. A filtration, $\mathscr{T}$, for $f$ on $M$ is a finite sequence of submanifolds with boundary, $\varnothing=M_{0} \subset M_{1} \subset \cdots \subset M_{k} \subset M_{k+1}=M$, satisfying:

(a) $f\left(t, M_{i}\right) \subset$ int $M_{i}$ for all $t>0$ and $0 \leqslant i \leqslant k+1$.

(b) $\cap_{t \in R} f\left(t, M_{i}-M_{i-1}\right)={ }_{\text {def }} L\left(M_{i}\right) \neq \varnothing$.

The limit set of the filtration is $L(\mathscr{N})={ }_{\text {def }} \cup_{i=1}^{k} L\left(M_{i}\right)$.

We say that one filtration, $\mathfrak{K}^{\prime}$, refines a second, $\mathfrak{K}$, if for every $M_{j}^{\prime} \in \mathfrak{R}^{\prime}$, there is $M_{j(i)} \in \mathscr{T}$ with $L\left(M_{j}^{\prime}\right) \subset L\left(M_{j(i)}\right)$.

5.5 Definition. For $x$ in $M$, the chain limit set of $x$ (with respect to $f$ ), is $C L(x)=\{y \mid$ for each $\varepsilon>0$, there is an $(\varepsilon, 1)$-chain from $x$ through $y\}$. See [C]. Similarly, for $\varepsilon>0$, define $C L(x, \varepsilon)$ to be the set of all points $y$ in $M$ such that there is an $(\varepsilon, 1)$-chain from $x$ through $y$. This is not exactly the same definition as the one used by Conley [C]; his definition results in a slightly smaller set. More important in the current context is the difference between these sets and the sets $Z(x)$ and $Z(x, \varepsilon)$ defined in 4.1 and 4.3. For example, if $p$ is a fixed saddle for $f$, and is isolated in $C R(f)$, then $Z(p)=\{p\}$, but $C L(p)$ contains the unstable manifold of $p$ as well (and more).

5.6 LemmA. Suppose $\left\{X_{i}\right\}, i=1,2, \ldots, n$, is a collection of pairwise disjoint, closed, nonempty, $f$-invariant sets whose union contains $C R(f)$. If we say $X_{i} \leqslant X_{j}$ if and only if $X_{i} \subset C L\left(X_{j}\right)$, then $\leqslant$ defines a partial ordering of $\left\{X_{i}\right\}$ and so can be extended to a linear ordering.

Proof. If both $X_{i} \subset C L\left(X_{j}\right)$ and $X_{j} \subset C L\left(X_{i}\right)$, then either $i=j$, or $X_{i} \cup X_{j} \subset$ $C R(f)$. If $i \neq j$, it is easy to see that whenever a chain component meets either $X_{i}$ or $X_{j}$, then it is contained in their intersection. As this intersection is assumed to be empty, it follows that $i=j$ and so the relation $\leqslant$ is antisymmetric. It is clearly transitive, and is reflexive since we are looking at chains "through" points rather 
than "to" points. (See 2.1 for the definition.) Thus $\leqslant$ defines a partial ordering. As there are only finitely many $X_{i}$ 's in the ordering, extending to a total ordering is a standard argument which we do not reproduce here.

We now state a result of Conley in the language of filtrations. (See [C, Chapter I, §8].)

5.7 THEOREM. Given a flow on $M$, there is a sequence of filtrations, $\mathfrak{T}_{j}=\left\{M_{j}^{i} \mid\right.$ $0 \leqslant i \leqslant k(j)+1\}$, such that $\cap_{j} L\left(\Re_{j}\right)=C R(f)$ and $\Re_{j+1}$ refines $\mathfrak{M}_{j}$ for each $j$.

C. Sufficient conditions for the existence of nontrivial quasi-attractors.

5.8 THEOREM. The following are equivalent:

(a) $f$ has an infinite number of attractors.

(b) $f$ has an infinite number of chain components.

(c) $f$ has a quasi-attractor that is not an attractor.

Proof. (c) implies (a) by 3.5 .

To see that (a) implies (b), note that every attractor meets $C R(f)$ and is locally isolated in $C R(f)$ (since $C R(f) \cap B(A)=C R(f) \cap A)$, so an infinite number of attractors implies an infinite number of chain components.

Finally we show that (b) implies (c). Let $\mathfrak{N}_{j}$ be the sequence of filtrations constructed in 5.7; as before denote $\Re_{j}=\left\{M_{j}^{i}\right\}$. As $C R(f) \subset \cup_{i} L\left(M_{j}^{i}\right)$, and there are only finitely many $M_{j}^{i}$,s for a given $j$, we see that one of the $M_{j}^{i}$,s must contain an infinite number of chain components. Hence we proceed inductively, à la Bolzano-Weierstrass. Choose $M_{1}^{*} \in \mathfrak{N}_{1}$ such that $L\left(M_{1}^{*}\right)$ contains infinitely many chain components and such that if $L\left(M_{1}^{i}\right)$ also contains infinitely many chain components, then $M_{1}^{*} \subset M_{1}^{i}$. Given $M_{j}^{*} \in \mathfrak{T}_{j}$, choose $M_{j+1}^{*} \in \mathfrak{R}_{j+1}$ with $M_{j+1}^{*}$ $\subset M_{j}^{*}$, infinitely many chain components in $L\left(M_{j+1}^{*}\right)$ and $M_{j+1}^{*}$ minimal among all $M_{j+1}^{i}$ that satisfy these first two properties. Now recall that if we define $A_{j}^{*}=$ $\cap_{t>0} f\left(t, M_{j}^{*}\right)$, then $A_{j}^{*}$ is an attractor of $f$; moreover, $A_{j+1}^{*} \subset A_{j}^{*}$ for all $j$. In fact there is a subsequence of $\left\{A_{j}^{*}\right\}$ for which strict inclusion holds, that is, $A_{j}^{*} \neq A_{j+1}^{*}$. (This is because any two chain components are separated by $\mathfrak{R}_{j}$ for all large $j$.) Now 3.5 applies to show that $\cap_{j} A_{j}^{*}$ is a quasi-attractor that is not an attractor.

5.9 REMARK. A. Lopes has some results in the direction of showing that a structurally stable system has only finitely many attractors. In particular, he shows that such a system has only finitely many hyperbolic attractors, and from this concludes that if the dimension of $M$ is two, then there are only finitely many attractors and hence no nontrivial quasi-attractors. See [Lo].

6. Density of basins. In this section we discuss sufficient conditions for the density of the basins of the chain transitive quasi-attractors. The most general of these conditions is the generalized saddle property, which says that a chain transitive invariant set that contains the omega limit set of an open set must be a quasi-attractor. The choice of the name of this property is motivated by the analogous situation for hyperbolic fixed points: if $p$ is a hyperbolic fixed point whose stable manifold has interior, then $p$ is a sink. In particular, the following type of behavior is ruled out. 


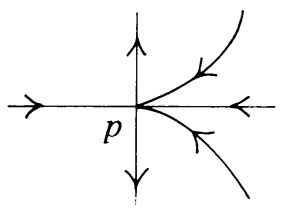

Figure 6.0

6.1 Definition. We say $f$ satisfies the generalized saddle property if whenever $X$ is a compact, $f$-invariant set with int $\{x \in M \mid \omega(x) \cap X \neq \varnothing\} \neq \varnothing$, then there is a quasi-attractor $A \subset X$. (For completeness, one should also require that $X$ contain a quasi-repellor any time int $\{x \in M \mid \alpha(x) \subset X\} \neq \varnothing$, but such a requirement is not necessary for what follows.)

6.2 LeMMA. Let $X=\bigcap_{n} A_{n}$ be a chain transitive quasi-attractor and let $\varepsilon>0$ be given. Then there is a value $n_{0}$ such that if $Y=\cap_{n=1}^{n_{0}} A_{n}$, then $Y$ is an $(\varepsilon, 1)$-chain transitive attractor.

Proof. Any such $Y$ is an attractor by 1.1. Now let $x \in X$ and consider $Z(x, \varepsilon)$. This set is $(\varepsilon, 1)$-chain transitive by definition (see 4.3), and is a neighborhood of $Z(x)=X$ by 4.4. Because $\cap_{n=1}^{k} A_{n}$ approaches $X$ in the Hausdorff topology as $k$ goes to infinity, we can find $n_{0}$ to satisfy $\cap_{n=1}^{n_{0}} A_{n} \subset Z(x, \varepsilon)$ and so $Y$ will be $(\varepsilon, 1)$-chain transitive as required.

6.3 Definition. Given a flow $f$, let $D(f)$ denote the union of the basins of all the chain transitive quasi-attractors of $f$. In other words, $D(f)=\{x \mid \omega(x)$ is contained in a chain transitive quasi-attractor of $f\}$. For $\varepsilon>0$, let $D_{\varepsilon}(f)$ denote the union of the basins of the $(\varepsilon, 1)$-chain transitive attractors.

6.4 REMARKS. (i) Every $(\varepsilon, 1)$-chain transitive quasi-attractor is contained in an $(\varepsilon, 1)$-chain transitive attractor, so we lose nothing by considering only attractors in the definition of $D_{\varepsilon}(f)$; in particular, $D_{\varepsilon}(f)$ is open.

(ii) $\cap_{e>0} D_{e}(f)=D(f)$.

(iii) If $f$ is an Axiom A flow, then $\operatorname{clos}\left(D_{\varepsilon}(f)\right)=M$ for all $\varepsilon>0$.

Proof. (i) follows from 6.2. To establish (ii), it suffices to show that $\cap D_{1 / n}(f) \subset$ $D(f)$. If $x \in \cap D_{1 / n}(f)$, then for each $n$ there is a $(1 / n, 1)$-chain transitive attractor $A_{n}$ with $\omega(x) \subset A_{n}$. Hence $\omega(x) \subset \cap A_{n}$ which is clearly a chain transitive quasi-attractor, so that $x \in D(f)$. (iii) follows from 5.5 and 5.6 of [BR].

6.5 THEOREM. The following are equivalent:

(a) $f$ satisfies the generalized saddle property.

(b) $D(f)$ is dense in $M$.

(c) $D(f)$ is residual in $M$.

Proof. That (c) implies (b) implies (a) is trivial. We shall complete the proof by showing that (a) implies (c). We claim that each $D_{1 / n}(f)$ is dense in $M$. Putting off the proof of this claim for the moment, we use it to prove the theorem. As each $D_{1 / n}(f)$ is open and dense, $\cap D_{1 / n}(f)$ is residual in $M$, and, by 6.4(ii), so is $D(f)$.

Proof OF THE ClaIM. If some $D_{1 / n}(f)$ were not dense, then its complement is closed, invariant and has nonempty interior. Denote the complement by $X_{n}$. By the 
generalized saddle property, there is a quasi-attractor in $X_{n}$; using minimality (see 3.3) we see that there is a chain transitive quasi-attractor $Y_{n}$ in $X_{n}$. Applying the last lemma for $\varepsilon \in(0,1 / n)$, we get a $(1 / n, 1)$-chain transitive attractor that contains $Y_{n}$ and so intersects $X_{n}=M-D_{1 / n}(f)$. This is absurd, and so we have the proof of the claim and thus the theorem.

6.6 COROLLARY. If $f$ satisfies the generalized saddle property and every quasiattractor of $f$ is an attractor, then $f$ has a finite number of chain transitive attractors whose basins are dense in $M$.

Proof. 5.8 shows that if every quasi-attractor of $f$ is an attractor, then $f$ has only finitely many attractors.

The proof of the theorem shows that if we are only concerned with $(\varepsilon, 1)$-chain transitive attractors for a given $\varepsilon>0$, then we can conclude that their basins are dense in $M$, since we have shown that each $D_{\varepsilon}(f)$ is open and dense.

6.7 COROLlaRY. If $f$ satisfies the generalized saddle property and $\varepsilon>0$, then $f$ has a finite number of $(\varepsilon, 1)$-chain transitive attractors whose basins form an open, dense subset of $M$.

6.8 Corollary. Suppose $f$ satisfies the following property: Whenever $X$ is $a$ compact invariant set that meets the omega limit set of every point in an open set in $M$, then there is a chain transitive quasi-attractor of $f, A$, such that $A \cap X \neq \varnothing$. Then $D(f)$ is dense in $M$.

Proof. The property just described is precisely what we used the generalized saddle property to obtain in the proof of 6.5 .

Next we are going to give the proof of Theorem C. First we establish a few lemmas that are necessary for this proof.

6.9 LemMa. Suppose $A$ is compact, invariant, and $(\varepsilon, 1)$-chain transitive. Then there exist constants $\varepsilon^{\prime}<\varepsilon$ and $\alpha>0$ such that $U_{\alpha}(A)$ is $\left(\varepsilon^{\prime}, 1\right)$-chain transitive.

Proof. We begin by showing that there is an $\varepsilon^{\prime}<\varepsilon$ such that $A$ is $\left(\varepsilon^{\prime}, 1\right)$-chain transitive.

(i) Choose $\delta>0$ such that $d(x, y)<\delta$ and $|t|<1$ imply that $d(f(t, x), f(t, y))<\frac{1}{2} \varepsilon$.

(ii) Choose a finite, $\delta$-dense subset of $A, X$.

(iii) Choose a finite $(\varepsilon, 1)$-chain $\left\{\left(x_{i}, t_{i}\right) \mid i=0,1, \ldots, p\right\}$ satisfying $x_{0}=x_{p}$ and $X \subset\left\{x_{i}\right\}$.

(iv) Let $\varepsilon_{0}=\max \left\{\frac{1}{2} \varepsilon, d\left(f\left(t_{i}, x_{i}\right), x_{i+1}\right)\right\}$, where $i$ goes from 0 to $p-1$. By the definition of an $(\varepsilon, 1)$-chain, it is clear that $\varepsilon_{0}<\varepsilon$, so define $\varepsilon^{\prime}=\frac{1}{2}\left(\varepsilon+\varepsilon_{0}\right)$. Now, if $y$ and $z$ are in $A$, we can choose $x_{j}$ and $x_{k}$ in $X$ to satisfy $d\left(x_{j}, f(1, y)\right)<\delta$ and $d\left(x_{k}, f(-1, z)\right)<\delta$. Then: $y \rightarrow x_{j} \rightarrow \cdots \rightarrow x_{k} \rightarrow z$ is an $\left(\varepsilon^{\prime}, 1\right)$-chain, where the chain from $x_{j}$ to $x_{k}$ is obtained from the periodic $\left(\varepsilon^{\prime}, 1\right)$-chain described in (iii). Thus $A$ is $\left(\varepsilon^{\prime}, 1\right)$-chain transitive.

(v) Finally, by arguing as in part (i), we can choose $\alpha>0$ such that $d(x, y)<\alpha$ and $|t| \leqslant 1$ imply $d(f(t, x), f(t, y))<\varepsilon^{\prime}$. It follows immediately that $U_{\alpha}(A)$ is $\left(\varepsilon^{\prime}, 1\right)$-chain transitive. 
6.10 LEMMA. The maps $F^{r}(M) \rightarrow F M(r \geqslant 0)$ defined by $f \rightarrow \operatorname{clos}\left(D_{\varepsilon}(f)\right)(\varepsilon>0)$ are all lower semicontinuous.

Proof. Suppose $A$ is an $(\varepsilon, 1)$-chain transitive attractor of $f$. By 6.9 , there are constants $\varepsilon^{\prime}<\varepsilon$ and an arbitrarily small $\alpha>0$ such that $U_{\alpha}(A)$ is $\left(\varepsilon^{\prime}, 1\right)$-chain transitive for $f$. Now, if $g$ is $C^{r}$ close enough to $f$, we have

(i) $g$ has an attractor $A(g)$ with $A(g) \subset U_{\alpha}(A)$ and $B(A) \subset U_{\alpha}(B(A(g)))$.

(ii) $U_{\alpha}(A)$ is $(\varepsilon, 1)$-chain transitive for $g$.

It follows that $A(g)$ is an $(\varepsilon, 1)$-chain transitive attractor for $g$, and so that $B(A(g)) \subset D_{\varepsilon}(g)$.

From the compactness of $M$ and the fact that $f$ has at most countably many attractors, we see that there are finitely many attractors of $f, A_{1}, \ldots, A_{k}$, such that $D_{\varepsilon}(f) \subset U_{\alpha}\left(\cup_{\alpha=1}^{k} B\left(A_{j}\right)\right)$. Applying the preceding argument to each of the $A_{j}$ 's, we see that if $g$ is sufficiently close to $f$, then $g$ has attractors $A_{j}(g)$, each $(\varepsilon, 1)$-chain transitive, and $B\left(A_{j}\right) \subset U_{\alpha}\left(B\left(A_{j}(g)\right)\right)$. Hence,

$$
D_{\varepsilon}(f) \subset U_{\alpha}\left(\cup B\left(A_{j}\right)\right) \subset U_{2 \alpha}\left(\cup B\left(A_{j}(g)\right)\right) \subset U_{3 \alpha}\left(D_{\varepsilon}(g)\right),
$$

and so $\operatorname{clos}\left(D_{\varepsilon}(f)\right) \subset U_{4 \alpha}\left(\operatorname{clos}\left(D_{\varepsilon}(g)\right)\right)$. As $\alpha$ can be taken arbitrarily small, this proves the lemma.

6.11 Definition. Let $H=\bigcap_{n}$ \{continuity points of $\operatorname{clos}\left(D_{1 / n}\left({ }_{-}\right)\right)$\}, so that $H$ is residual.

6.12 THEOREM. $C^{0}$ generically the basins of the chain transitive quasi-attractors are residual in $M$.

Proof. By 6.11 it suffices to show that $D(f)$ is residual in $M$ for $f$ in $H$. By [O] we can choose Axiom A flows $f_{k}$ with $f_{k}$ converging to $f$ in the $C^{0}$ topology. By 6.4(iii), $\operatorname{clos}\left(D_{1 / n}\left(f_{k}\right)\right)=M$ for all $n$ and all $k$. As $f$ is assumed to be in $H$, $\operatorname{clos}\left(D_{1 / n}(f)\right)=M$ for all $n$. Thus $D_{1 / n}(f)$ is open and dense in $M$, so $\cap_{n} D_{1 / n}(f)$ is residual in $M$. That $\cap D_{1 / n}(f)=D(f)$ was established in 6.4(ii).

6.13 REMARK. $C^{0}$ generically a flow has quasi-attractors other than attractors.

Proof. Combine parts (d) and (h) of Theorem 1 of [PP].

Next we describe a property of flows that is stronger than the generalized saddle property, but one that has been the object of some attention in the study of dynamical systems.

6.14 Definition. We say $f$ has the weak shadowing property if: Whenever $x, y$ are in $M$ and for each $\varepsilon>0$ there is an $(\varepsilon, 1)$-chain from $x$ through $y$, then there are sequences $x_{n} \rightarrow x, y_{n} \rightarrow y$, and $t_{n} \geqslant 0$ such that $f\left(t_{n}, x_{n}\right)=y_{n}$. In other words, given any $x$ in $M$ and $y \in C L(x)$, there are orbit segments that go from arbitrarily near $x$ to arbitrarily near $y$. $(C L(x)$ was defined in 5.5.)

6.15 REMARK. The usual shadowing property that has been studied in the past is defined as follows: Given any $\delta>0$, there is a value $\varepsilon>0$ such that any $(\varepsilon, 1)$-chain can be $\delta$-shadowed; that is, there is an actual $f$-orbit that traces it within $\delta$. (See [B1], for example.) The chief difference in the definitions of the two shadowing properties is that the weak shadowing property is concerned only with tracing from $x$ to $y$ when one can get from $x$ to $y$ via an $(\varepsilon, 1)$-chain for any $\varepsilon>0$ 
and asserts nothing about tracing of arbitrary $(\varepsilon, 1)$-chains for any particular $\varepsilon>0$. To what degree the differences in the two definitions are cosmetic rather than real is of no real concern here, and we shall say nothing more about it.

6.16 COROLLARY. If $f$ has the weak shadowing property, then it has the variant of the generalized saddle property described in 6.8 , and so $D(f)$ is dense.

Proof. Suppose $f$ has shadowing and $X$ is a compact, $f$-invariant set that contains the omega limit set of every point in $U$, where $U$ is open in $M$. For $\varepsilon>0$ consider the collection of $Z(x, \varepsilon)$ 's for $x$ in $U$. By 4.4(iii) this is a finite collection. It inherits a total ordering from 5.6. If the minimal element under this ordering is not an attractor, then we get a contradiction to shadowing by using the arguments of 5.3 as follows: Let $Z(x, \varepsilon)$ be this minimal element, where $x \in U$; if $Z(x, \varepsilon)$ is not an attractor then there is an $(\varepsilon, 1)$-chain component $Y$ that intersects $C(x)-$ $Z(x, \varepsilon)$. By the definition of the ordering $Y \cap X=\varnothing$. One can show that no orbit goes from a compact neighborhood of $x$ in $U$ to within a small neighborhood of $Y$. As we have shown that $Y \cap C L(x) \neq \varnothing$ we thereby contradict the weak shadowing property. Thus $Z(x, \varepsilon)$ must be an attractor. It is clear from the definition of the ordering that if $\varepsilon^{\prime}$ is smaller than $\varepsilon$, and $Z\left(x^{\prime}, \varepsilon^{\prime}\right)$ is the corresponding minimal element of $\left\{Z\left(y, \varepsilon^{\prime}\right) \mid y \in U\right\}$, then $Z\left(x^{\prime}, \varepsilon^{\prime}\right) \subset Z(x, \varepsilon)$. It follows that the intersection of these minimal elements for all $\varepsilon>0$ is nonvoid and intersects $X$; clearly this intersection is a chain transitive quasi-attractor. Thus the property described in 6.8 holds for $f$ and so we conclude that $D(f)$ is dense.

6.17 REMARK. The use of the basins of the chain transitive quasi-attractors instead of the basins of the chain transitive attractors is necessary in 6.16 as Example 3.2 shows. As this example also satisfies the generalized saddle property we see that the consideration of quasi-attractors is necessary in 6.5 as well.

7. Persistence of attractors. As mentioned in the introduction, we would like the attractors that we are studying to be "stable" under perturbations of the flow. That is, given an attractor $A$ for $f$, and a flow $g$ near $f$, we want to know that $g$ has an attractor $A(g)$ that is near $A$ in some sense. In what follows we describe a weak form of stability, but one that is possessed by a $C^{r}$ generic set of flows for every $r \geqslant 0$.

7.1 Definition. An attractor $A$ for $f$ is called $C^{r}$-persistent if whenever $f_{n}$ is a sequence of flows converging to $f$ in the $C^{r}$ topology, then each $f_{n}$ has an attractor $A_{n}$ and $A_{n}$ approaches $A$ in the Hausdorff topology. Recall that we denote the $C^{r}$ metric on $F^{r}(M)$ by $d_{r}$, and the Hausdorff metric on $F M$ by $d_{H}$.

7.2 REMARK. This definition, and much of the work in this section, is related to the work of Takens on the tolerance stability conjecture. See [Ta1] and [Ta2].

7.3 Remark. Persistence is a relatively weak condition, much weaker than the notions of structural or topological stability described in the introduction. However, as mentioned above, it is a property that holds for all attractors of each flow in a $C^{r}$-residual subset of $F^{r}(M)$ for every $r \geqslant 0$, while these stronger properties do not. 
7.4 Remark. Persistence is not an open condition. For example, any point attractor is necessarily persistent, as we shall see; however, if the point is not hyperbolic, then there will be nearby maps whose corresponding attractors are not persistent. (Expand the sink to be a small attracting disk that is composed entirely of fixed points.)

7.5 Lemma. Let $\mathcal{P}$ denote the $C^{1}$-residual set of flows that are Kupka-Smale and have their periodic orbits dense in their nonwandering sets. (See $[\mathbf{P}],[\mathbf{S}]$ and $[\mathbf{N}]$.) Suppose $f \in \mathscr{P}$ and $A$ is a chain transitive attractor for $f$ with $A \subset \Omega(f)$. Then $A$ is persistent.

Proof. Let $H_{k}$ denote the set of periodic orbits of $f$ that are contained in $A$ and that persist under $C^{1}$ perturbations of size $1 / k$. (By this we mean that $\gamma$ is in $H_{k}$ if there is a neighborhood, $N(\gamma)$, of $\gamma$ in $M$ such that whenever $g$ is $C^{1}-(1 / k)$-close to $f$, then there is a homeomorphism $h$, from $N(\gamma)$ into $M$, such that $h$ is $C^{0}-(1 / k)$-close to the inclusion and such that $f=h^{-1} \circ g \circ h$ on $N(\gamma)$.) Then $H_{k} \subset H_{k+1}$ for all $k$, and each periodic orbit of $f$ in $A$ is contained in one of the $H_{k}$ 's. (This is Hartman's theorem; see [H] or [N].) Now suppose $f_{n}$ approaches $f$ in the $C^{1}$ topology. Let $U$ be open in $M$ and satisfy $A \subset U \subset \operatorname{clos}(U) \subset B(A)$ and $f(1, \operatorname{clos}(U)) \subset U$. As we shall describe in detail in 7.7, it is possible to pick $n_{0}$ such that whenever $n \geqslant n_{0}$, there is an attractor $A_{n}=\bigcap_{t>0} f_{n}(t, \operatorname{clos}(U))$ of $f_{n}$. Let $H_{k}^{(n)}$ be the periodic orbits of $f_{n}$ corresponding to orbits in $H_{k}$. Note that $H_{k}^{(n)}$ approaches $H_{k}$ in the Hausdorff topology as $n$ goes to infinity. Moreover, for each value of $k=1,2,3, \ldots$, there is an $n_{k}$ such that $H_{k}^{\left(n_{k}\right)} \subset A_{n}$. Thus for each $k$ we have

$$
H_{k}=\lim _{n} H_{k}^{(n)}=\lim _{n} \inf H_{k}^{(n)} \subset \lim \inf A_{n},
$$

and hence $\cup_{k} H_{k} \subset \lim \inf A_{n}$. Finally, because lim $\inf A_{n}$ is closed, it contains $\operatorname{clos}\left(\cup_{k} H_{k}\right)=A$. It is not hard to see that $A \supset \lim \sup A_{n}$ (we prove this below in 7.7). Therefore $\lim A_{n}$ exists and is equal to $A$ (taking this limit in the Hausdorff topology on $F M$ ).

7.6 REMARK. If $f$ is Kupka-Smale, the periodic orbits of $f$ are dense in $\Omega(f)$, and $A$ is a Thom attractor for $f$ (an attractor with a dense orbit), then $A$ is chain transitive, and persistent by 7.5 .

7.7 Lemma. Suppose $A$ is an attractor of $f$, and $f_{n} \rightarrow f C^{r}$. Let $U$ be an open set in $M$ with $A \subset U \subset \operatorname{clos}(U) \subset B(A)$. Then there is an $n_{0}$ such that for $n \geqslant n_{0}, f_{n}$ has an attractor $A_{n}$ with $A_{n} \subset U \subset \operatorname{clos}(U) \subset B\left(A_{n}\right)$. Moreover, $\lim \sup A_{n} \subset A$ and $B(A) \subset \lim \inf B\left(A_{n}\right)$.

Proof. Let $\varepsilon>0$ be small enough that $U_{e}(A) \subset U$. Using Lemma 1.6 we can choose an open set $G \subset U_{\varepsilon}(A)$ that is forward invariant and satisfies $f(1, \operatorname{clos}(G)$ ) $\subset G$. Then there is an integer $n_{1}$ such that $n \geqslant n_{1}$ implies that $f_{n}(1, \operatorname{clos}(G)) \subset G$ as well. Define $V_{n}=f_{n}([0,1], G)$ and $V_{n}^{\prime}=f_{n}([0,1], \operatorname{clos}(G))$. Clearly $V_{n}$ is open, $V_{n}^{\prime}$ is closed, and $V_{n} \subset V_{n}^{\prime}$ for all $n$; in fact, $\operatorname{clos}\left(V_{n}\right)=V_{n}^{\prime}$. In addition, we have

(A) $f_{n}\left(1, V_{n}^{\prime}\right) \subset V_{n}$ for $n \geqslant n_{1}$.

(B) $f_{n}\left(t, V_{n}\right) \subset V_{n}$ for all $t \geqslant 0$ and $n \geqslant n_{1}$. 
(C) $A_{n}={ }_{\text {def }} \cap_{t>0} f\left(t,\left(V_{n}^{\prime}\right)\right)$ is an attractor when $n>n_{1}$.

Thus far in the proof we have constructed attractors $A_{n}$ which for large $n$ satisfy $A_{n} \subset U_{\varepsilon}(A) \subset U$; by letting $\varepsilon$ go to 0 we see that $\lim \sup A_{n} \subset A$. It remains to show that $\operatorname{clos}(U) \subset B\left(A_{n}\right)$ for all large $n$ and that $B(A) \subset \lim \inf B\left(A_{n}\right)$. Note that the latter inclusion follows from the former as we can choose a sequence of open sets $U_{j} \subset B(A)$ whose closures converge to $\operatorname{clos}(B(A))$ in the Hausdorff topology.

The proof that $\operatorname{clos}(U) \subset B\left(A_{n}\right)$ for all large $n$ is also easy. Using Lemma 1.6 choose a subset $L$ of $U$ that is open and forward invariant. The lemma also gives us a constant $T(L) \geqslant 0$ such that $f(t, \operatorname{clos}(U)) \subset L$ for $t \geqslant T(L)$. As in the first part of this proof, we can find $n_{2} \geqslant 0$ such that $n \geqslant n_{2}$ implies that $f_{n}$ has an attractor $A_{n}$ with $A_{n} \subset L \subset B\left(A_{n}\right)$. There is also a constant $n_{3}$, depending upon $T(L)$, such that $f_{n}(T(L), \operatorname{clos}(U)) \subset G \subset B\left(A_{n}\right)$ for $n \geqslant \max \left(n_{2}, n_{3}\right)$. As $B\left(A_{n}\right)$ is invariant under $f_{n}$, we conclude that $\operatorname{clos}(U) \subset B\left(A_{n}\right)$ for all large $n$.

7.8 Definition. Suppose that $U$ is a nonempty, open subset of $M$. Let $g_{U}$ be a map from $F^{r}(M)$ into $F M$ defined by

$$
g_{U}(f)= \begin{cases}M & \text { if no attractor } A \text { of } f \text { satisfies } \\ & A \subset U \subset \operatorname{clos}(U) \subset B(A), \\ A & \text { if } A \text { is an attractor of } f \text { with } \\ & A \subset U \subset \operatorname{clos}(U) \subset B(A) .\end{cases}
$$

7.9 Lemma. Each map $g_{U}$ is upper semicontinuous.

Proof. Suppose $f_{n} \rightarrow f$. If $g_{U}(f)=M$, then upper semicontinuity at $f$ is trivial. If $g_{U}(f)=A \neq M$, then using 7.7 we see that for all large $n, g_{U}\left(f_{n}\right)=A_{n}$ and $\lim \sup A_{n} \subset A$.

7.10 LEMma. There is a countable family, Q that if $K$ and $K^{\prime}$ are disjoint closed subsets of $M$, then there is a $U$ in $थ$ with $K \subset U$ and $\operatorname{clos}(U) \cap K^{\prime}=\varnothing$.

Proof. This is no more than the fact that a compact manifold has a countable basis for its topology.

7.11 TheOREM. There is a residual set $C$ in the set of $C^{r}$ flows in the $C^{r}$ topology $(r \geqslant 0)$ such that $f \in C$ implies that every attractor of $f$ is persistent.

Proof. Let $\mathscr{Q}=\left\{U_{i}\right\}_{i=1}^{\infty}$ be the family of open sets of 7.10 , ordered in some way. By 7.9 and 1.9 if we let $C_{i}$ denote the set of continuity points of $g_{U_{i}}$, then each $C_{i}$ is residual in $F^{r}(M)$ and therefore $C==_{\text {def }} \cap_{i} C_{i}$ is also residual.

It remains to show that if $f$ is in $C$ and $A$ is an attractor for $f$, then $A$ is persistent. We will accomplish this by showing that if $f$ has a nonpersistent attractor $A$, then $f \notin C_{n}$ for some $n$. We proceed as follows: As $A$ and $M-B(A)$ are closed and disjoint, 7.10 shows that we can choose some $U_{j}$ in $थ$ with $A \subset U_{j} \subset \operatorname{clos}\left(U_{j}\right) \subset$ $B(A)$. If $A$ is not persistent, then there is a sequence $f_{n}$ approaching $f$ such that each $f_{n}$ has an attractor $A_{n}$, and these attractors satisfy

(i) $A_{n} \subset U_{j} \subset \operatorname{clos}\left(U_{j}\right) \subset B\left(A_{n}\right)$ for all $n$, and 
(ii) $\left\{A_{n}\right\}$ converges to $X$ in the Hausdorff topology, where $X$ is a proper subset of A.

Thus $g_{U_{j}}\left(f_{n}\right)=A_{n}$ and $\lim _{n} g_{U_{j}}\left(f_{n}\right)=X \neq A=g_{U_{j}}(f)$, and so $f \notin C_{j}$.

7.12 THEOREM. There is a residual subset $C_{r}^{*}$ of $F^{r}(M)$ for each $r \geqslant 0$, such that if $f \in C_{r}^{*}, A$ is an attractor for $f$, and $f_{n} \rightarrow f$ in $F^{r}(M)$, then there are attractors $A_{n}$ for $f_{n}$ with $A_{n} \rightarrow A$ and $B\left(A_{n}\right)$ approaches $B(A)$ in the Hausdorff topology.

Proof. Let $C$ be the set described in 7.11, and define $C_{r}^{*}$ by $C_{r}^{*}=\{f \mid f \in C$ and $\left.f^{*} \in C\right\}$, where $f^{*}$ is the flow we get from $f$ by reversing the time parametrization $\left(f^{*}(t, x)=f(-t, x)\right.$; see 1.7). The theorem follows once we note that if $A$ is any attractor of $f$, then $M-B(A)$ is an attractor of $f^{*}$, so (using 7.7) we see that $M-B\left(A_{n}\right)$ converges to $M-B(A)$. As remarked in 1.C this is equivalent to the convergence of $B\left(A_{n}\right)$ to $B(A)$, in the sense that $\lim _{n} d_{H}\left(B\left(A_{n}\right), B(A)\right)=0$.

7.13 Corollary. If $f \in C_{r}^{*},\left\{f_{n}\right\}_{n=1}^{\infty}$ is a sequence in $F^{r}(M)$ with $f_{n} \rightarrow f$, and $X$ is a quasi-attractor for $f$, then each $f_{n}$ has a quasi-attractor $X_{n}$ such that $X_{n} \rightarrow X$.

Proof. Let $X=\bigcap_{k} A_{k}$; we can assume $d_{H}\left(X, A_{k}\right)<1 / k$ for each $k$. As $f \in C_{r}^{*}$, for each $k$ there is a sequence of attractors $A_{k}^{(n)} \rightarrow A_{k}$. Hence for each $k$ there is a constant $N(k)$ such that if $n \geqslant N(k)$, then $d_{H}\left(A_{k}^{(n)}, A_{k}\right)<1 / k$. We can (and do) assume that $N(k+1)>N(k)$ for each $k$. Let $X_{n}=A_{K(n)}^{(n)}$ where

$$
K(n)= \begin{cases}\sup \{k \mid N(k) \leqslant n<N(k+1)\} & \text { for } n \geqslant N(1), \\ 1 & \text { for } n<N(1) .\end{cases}
$$

Now we complete the proof by showing $X_{n} \rightarrow X$. The definitions ensure that $n \geqslant N(K(n))$ for each $n \geqslant n(1)$, and so

$$
\begin{aligned}
d_{H}\left(X_{n}, X\right) & \leqslant d_{H}\left(X_{n}, A_{K(n)}\right)+d_{H}\left(A_{K(n)}, X\right) \\
& <d_{H}\left(A_{K(n)}^{n}, A_{K(n)}\right)+1 / K(n)<2 / K(n) \rightarrow 0
\end{aligned}
$$

as $n \rightarrow \infty$. The convergence of the basins is similar.

Thus far we have shown that, generically, attractors and quasi-attractors persist. However, the main objects of concern are the chain transitive attractors and quasi-attractors. We would like to know that they persist as chain recurrent quasi-attractors. We shall in fact prove this in 7.15 and 7.20. We begin with a simple observation.

7.14 REMARK. Let $X$ be a compact, nonempty, $f$-invariant set that contains every chain component that it meets (for example, let $X$ be an attractor). Then $X$ is chain transitive if and only if it contains only one chain component.

7.15 THEOREM. For any $r \geqslant 0$, there is a residual subset $T_{r}$ of $F^{r}(M)$ with the property that if $f \in T_{r}$ and $A$ is a chain transitive attractor for $f$, then each sufficiently nearby flow $g$ has a chain transitive attractor $A_{g}$ such that $A_{g}$ approaches $A$ and $B\left(A_{g}\right)$ approaches $B(A)$ in the Hausdorff topology as $g$ approaches $f C^{r}$.

Proof. Given an open set $U$ in $M$ and $\varepsilon>0$, let $N_{U, e}(f)$ be the number of distinct $(\varepsilon, 1)$-chain components of $f$ that meet $U$. Note that $N_{U, \boldsymbol{e}}$ is finite valued; 
see 4.4(iii) for example. Now we claim that:

As a map into the reals, $N_{U, \varepsilon}$ is lower semicontinuous; that is, $\lim _{g \rightarrow f} \inf N_{U, \varepsilon}(g) \geqslant N_{U, \varepsilon}(f)$.

Before proving the claim, we use it to finish the proof of the lemma. Note that the proof would be easy if we could deal directly with chain components instead of with $(\varepsilon, 1)$-chain components; however, if we were to make this change, the corresponding version of the claim would be false. Let $T_{r}^{\prime}=\cap_{U \in \mathcal{Q}} \cap_{k=1}^{\infty}\{f \mid f$ is a continuity point of $N_{U, 1 / k}$, where $\mathcal{U}$ is as in 7.10. Now let $T_{r}=T_{r}^{\prime} \cap C_{r}^{*}$, where $C_{r}^{*}$ is the residual set described in 7.12. Suppose $f$ is in $T_{r}$ and $A$ is a chain transitive attractor of $f$. Choose $k$ large enough that the $(1 / k, 1)$-chain component of $f$ that contains $A$ is itself uniformly contained in $B(A)$. Call this $(1 / k, 1)$-chain component $X$. Recall that all $(1 / k, 1)$-chain components for $f$ are uniformly bounded apart, say by $\delta>0$. Choose $U \in \mathcal{U}$ such that $A \subset \operatorname{clos}(X) \subset U \subset \operatorname{clos}(U) \subset$ $U_{\delta}(X) \subset B(A)$. (This can be done as $\operatorname{clos}(X)$ and $M-U_{\delta}(X)$ are closed and disjoint.) Since $f \in C_{r}^{*}$, whenever $g$ is close enough to $f, g$ will have an attractor $A_{g}$ also satisfying $A_{g} \subset U \subset \operatorname{clos}(U) \subset B\left(A_{g}\right)$, with $A_{g} \rightarrow A$ and $B\left(A_{g}\right) \rightarrow B(A)$ as $g \rightarrow f$. Moreover, as we have ensured that $N_{U, 1 / k}(f)=1$ and that $f$ is a continuity point of $N_{U, 1 / k}$, we conclude that $N_{U, 1 / k}(g)=1$ for all $g$ sufficiently close to $f$. It now follows from 7.14 that $A_{g}$ is chain transitive.

Proof OF ClAIM. By compactness and the definition of an $(\varepsilon, 1)$-chain, if $X$ is a compact piece of an $(\varepsilon, 1)$-chain component of $f$, then there is a $\delta>0$ such that $X$ is itself $(\varepsilon-\delta, 1)$-chain transitive. (This is essentially the same as 6.9.) Thus if $g$ is close enough to $f, X$ will be $(\varepsilon, 1)$-chain transitive for $g$. Since $N_{U, e}(f)$ is finite, this argument shows that if $g$ is close enough to $f$, then $N_{U, e}(f)<N_{U, e}(g)$. (Any two $(\varepsilon, 1)$-chain components for $f$ fail to coalesce by a certain finite amount; this separation is preserved by all $g$ sufficiently close to $f$. To see this, let $\mathfrak{N}$ be a filtration for $f$ that separates all of the $(\varepsilon, 1)$-chain components; then $\mathscr{N}$ is a filtration for all $g$ near $f$, so these $(\varepsilon, 1)$-chain components do not coalesce for $g$ near $f$. This establishes the claim, and hence the lemma.)

Finally, we turn to the question of persistence of chain transitive quasi-attractors, beginning with several definitions.

7.16 Definition. We define three maps, $h, h_{1}$ and $h_{2}$ from $F^{r}(M) \times F M$ into $[0, \infty)$ as follows:

$h_{1}(f, X)=\inf \{\varepsilon>0 \mid X$ is contained in an $(\varepsilon, 1)$-chain transitive set $\}$.

$h_{2}(f, X)=d_{H}\left(X, Z\left(X, h_{1}(f, X)\right)\right)$, where $d_{H}$ is the Hausdorff metric on $F M$ and $Z(X, \varepsilon)=Z(x, \varepsilon)$ for any $x$ in $X$ (this set was defined in $\S 4$ ); we take $Z(X, 0)$ to mean $Z(x)$ for any $x$ in $X$. When we need to emphasize the dependence of $Z(X, \varepsilon)$ on $f$, we write $Z_{f}(X, \varepsilon)$.

$h(f, X)=h_{1}(f, X)+h_{2}(f, X)$.

7.17 REMARKs. (A) The definition of $h_{2}(f, X)$ is independent of the choice of the point $x$ in $X$ used to define $Z\left(X, h_{1}(f, X)\right)$. This is because $h_{1}(f, X)$ is big enough to ensure that all of $X$ is contained in a unique $\left(h_{1}(f, X), 1\right)$-chain component.

(B) $h(f, X)=0$ if and only if $X$ is a chain component of $f$. 
(C) $h$ is continuous if both $h_{1}$ and $h_{2}$ are.

Our plan is to first show that $h$ is continuous, then apply Takens' lemma (1.11) to get a residual subset of $F^{r}(M)$, and finally to show that chain transitive quasiattractors of flows in this residual set persist as chain transitive quasi-attractors.

\subsection{LEMMA. $h_{1}$ is continuous.}

Proof. Let $X, f$ and $\delta>0$ be given. Choose $\varepsilon>0$ to satisfy: $h_{1}(X, f)<\varepsilon<$ $h_{1}(X, f)+\delta$. Then there is an $(\varepsilon, 1)$-chain component of $f, Y$, with $X \subset Y$. Choose $\delta^{\prime}>0$ such that $d_{r}(f, g)<\delta^{\prime}$ implies $d(f(t, x), g(t, x))<\delta$ whenever $|t| \leqslant 2$. Then any $(\varepsilon, 1)$-chain for $f$ is an $(\varepsilon+\delta, 1)$-chain for $g$ for any $g$ with $d_{r}(f, g)<\delta^{\prime}$, and so $Y$ is $(\varepsilon+\delta, 1)$-chain transitive for $g$. Let $Y^{\prime}$ be the $(\varepsilon+2 \delta, 1)$-chain component of $g$ that contains $Y$. Then $Y^{\prime}$ is a neighborhood of $Y$, and so whenever $X^{\prime}$ is close enough to $X$ in the Hausdorff topology, then $X^{\prime} \subset Y^{\prime}$ and therefore $h_{1}\left(g, X^{\prime}\right) \leqslant \varepsilon$ $+2 \delta<h_{1}(f, X)+3 \delta$. Hence, if $\left(f_{n}, X_{n}\right) \rightarrow(f, X)$, then $\lim \sup h_{1}\left(f_{n}, X_{n}\right)<$ $h_{1}(f, X)$.

Now suppose $\left(f_{n}, X_{n}\right) \rightarrow(f, X)$ and $\lim h_{1}\left(f_{n}, X_{n}\right)=\varepsilon$. Let $\delta>0$ be given. Let $Y_{n}$ be the $(\varepsilon+\delta, 1)$-chain component of $f_{n}$ that contains $X_{n}$. Choose $\delta^{\prime}$ as in the first part of the proof. For $n$ large, $d_{r}\left(f, f_{n}\right)<\delta^{\prime}$, so $Y_{n}$ will be an $(\varepsilon+2 \delta, 1)$-chain transitive set for $f$; let $W_{n}$ be the $(\varepsilon+3 \delta, 1)$-chain component of $f$ that contains $Y_{n}$. As all $(\varepsilon+3 \delta, 1)$-chain components are uniformly bounded away from each other, we see that there is a value $n_{0}$ such that if $n, m$ are bigger than $n_{0}$ then $W_{n}=W_{m}$. Call this set $W$. Note that $X_{n} \subset Y_{n} \subset \operatorname{int}\left(W_{n}\right)=\operatorname{int}(W)$ for large $n$, so $X \subset$ $\operatorname{clos}(W)$. It follows that $X$ is contained in the $(\varepsilon+4 \delta, 1)$-chain component of $f$ that contains $W$; hence: $h_{1}(f, X) \leqslant \varepsilon+4 \delta=\lim h_{1}\left(f_{n}, X_{n}\right)+4 \delta$. It follows that for an arbitrary sequence $\left(f_{n}, X_{n}\right) \rightarrow(f, X)$, we have $h_{1}(f, X) \leqslant \lim \inf h_{1}\left(f_{n}, X_{n}\right)$. This and the result in the first paragraph of this proof combine to give the continuity of $h_{1}$ at every point.

\subsection{LEMMA. $h_{2}$ is continuous.}

Proof. Let $f, X$ and $\delta>0$ be given. Note that it suffices to show that the map $(f, X) \rightarrow Z\left(X, h_{1}(f, X)\right)$ is continuous. Define $\delta^{\prime}$ as in Lemma 7.18. Then $d_{r}(f, g)$ $<\delta^{\prime}$ implies that $Z\left(X, h_{1}(f, X)\right)$ is $\left(h_{1}(f, X)+\delta, 1\right)$-chain transitive for $g$; in other words, $Z_{f}\left(X, h_{1}(f, X)\right) \subset Z_{g}\left(X, h_{1}(f, X)+\delta\right)$. Also, by Lemma 7.18 , there is a constant $\delta_{0}>0$ such that if $d_{r}(f, g)<\delta_{0}$ and $d_{H}\left(X, X^{\prime}\right)<\delta_{0}$, then $h_{1}\left(g, X^{\prime}\right)+\delta$ $>h_{1}(f, X)$, and so $Z_{f}\left(X, h_{1}(f, X)\right) \subset Z_{g}\left(X, h_{1}\left(g, X^{\prime}\right)+2 \delta\right)$. In fact, using the continuity of $h_{1}$ and the fact that $Z_{g}\left(X, h_{1}\left(g, X^{\prime}\right)+2 \delta\right)$ contains a neighborhood of $X$ for all $X^{\prime}$ near enough to $X$, we conclude that for $d_{H}\left(X, X^{\prime}\right)<\delta_{1}$ (for some $\left.\delta_{1}>0\right)$, we have $Z_{f}\left(X, h_{1}(f, X)\right) \subset Z_{g}\left(X^{\prime}, h_{1}\left(g, X^{\prime}\right)+2 \delta\right)$.

Next note that for any $\varepsilon>0$ and $\delta$ small enough, we know that

$$
Z_{g}\left(X^{\prime}, h_{1}\left(g, X^{\prime}\right)+2 \delta\right) \subset U_{e}\left(Z_{g}\left(X^{\prime}, h_{1}\left(g, X^{\prime}\right)\right)\right) \text {. }
$$

Thus for any $\varepsilon>0$, we can choose $\delta, \delta_{0}, \delta_{1}$, and $\delta^{\prime}$ small enough that

$$
Z_{f}\left(X, h_{1}(f, X)\right) \subset U_{e}\left(Z_{g}\left(X^{\prime}, h_{1}\left(g, X^{\prime}\right)\right)\right)
$$

is true whenever $g$ and $X^{\prime}$ are sufficiently close to $f$ and $X$. 
It remains to show that we can reverse the places of $Z_{f}$ and $Z_{g}$ in (*). Suppose that $f_{n} \rightarrow f, X_{n} \rightarrow X, x_{n} \in Z_{f_{n}}\left(X_{n}, h_{1}\left(f_{n}, X_{n}\right)\right)$ and $x_{n} \rightarrow x$. Now let $\delta>0$ be given and choose $\delta^{\prime}$ as above. For $n \geqslant n_{0}, d_{r}\left(f_{n}, f\right)<\delta^{\prime}$, so $Z_{f_{n}}\left(X_{n}, h_{1}\left(f_{n}, X_{n}\right)\right)$ is $\left(h_{1}\left(f_{n}, X_{n}\right)+\delta, 1\right)$-chain transitive for $f$, and therefore, when $n$ is large enough, $\left(h_{1}(f, X)+2 \delta, 1\right)$-chain transitive for $f$. Thus $x_{n} \in Z_{f}\left(X, h_{1}(f, X)+3 \delta\right)$ for all large $n$ (this uses the fact that distinct $(\eta, 1)$-chain components are uniformly bounded apart whenever $\eta>0)$. Therefore, $x \in \operatorname{clos}\left(Z_{f}\left(X, h_{1}(f, X)+3 \delta\right)\right) \subset$ $Z_{f}\left(X, h_{1}(f, X)+4 \delta\right)$. As $\delta$ was arbitrary, $x \in Z_{f}\left(X, h_{1}(f, X)\right)$. It follows that if $\varepsilon>0$, there is a constant $n(\varepsilon)$ such that $n \geqslant n(\varepsilon)$ implies $Z_{f_{n}}\left(X_{n}, h_{1}\left(f_{n}, X_{n}\right)\right) \subset$ $U_{e}\left(Z_{f}\left(X, h_{1}(f, X)\right)\right)$, and so the proof is complete.

Now define a map $\bar{h}: F^{r}(M) \rightarrow F(F M)$ by $\bar{h}(f)=\{X \in F M \mid h(f, X)=0\}$. By 1.9 and 1.10 the set of continuity points of this map is a residual subset of $F^{r}(M)$. Let $H_{r}$ denote the intersection of this set with the set $C_{r}^{*}$ of 7.12 .

7.20 THEOREM. Suppose $f \in H_{r}, X$ is a chain transitive quasi-attractor of $f$, and $f_{n} \rightarrow f\left(C^{r}\right)$. Then each $f_{n}$ has a chain transitive quasi-attractor $X_{n}$ such that $X_{n} \rightarrow X$.

Proof. There are quasi-attractors $Y_{n}$ for $f_{n}$ with $Y_{n} \rightarrow X$ by 7.13. Each $Y_{n}$ contains a chain transitive quasi-attractor $X_{n}$ by 3.3. If $\lim \inf X_{n}=X$ we are done; if not, choose a subsequence, also called $X_{n}$, such that $\lim X_{n}=X^{\prime} \subsetneq X$. Clearly $X^{\prime}$ is not a chain component of $f$, so $X^{\prime} \notin \bar{h}(f)$ by 7.17(B). Thus there is an $\varepsilon>0$ such that $X^{\prime} \notin U H_{2 \varepsilon} \bar{h}(f)$, where $U H_{2 \varepsilon}$ denotes the $2 \varepsilon$-ball taken in the Hausdorff metric on $F(F M)$ induced by the metric $d_{H}$ on $F M$. For all large $n$, $d_{H}\left(X_{1}, X^{\prime}\right)<\varepsilon$, so $X_{n} \notin U H_{\varepsilon}(\bar{h}(f))$. As $X_{n}$ is in $\bar{h}\left(f_{n}\right)$ by assumption, we have $\bar{h}\left(f_{n}\right) \not \subset H_{e}(\bar{h}(f))$ for all large $n$. This contradicts the assumption that $f \in H_{r}$, and so proves the theorem.

7.21 Proof of Theorem A. Combine 7.12, 7.13, 7.15, and 7.20.

8. Conclusion. There are several interesting questions that remain unanswered. Primary among these are the following two.

Is the generalized saddle property generic?

Generically, is every chain transitive quasi-attractor an attractor?

The first of these questions has been discussed throughout this paper, the second has not. Regarding it, we have shown that "every quasi-attractor is an attractor" is not a generic property by using Newhouse's examples. However, it appears that the chain transitive quasi-attractors in these examples are periodic sinks. It would be interesting (even surprising) to have a proof that this is essentially the generic case; it would also be interesting to have a counterexample. Relevant here are the examples of Bowen, Franks, and Young, which are examples of Kupka-Smale diffeomorphisms of the disk with nontrivial quasi-attractors. These examples do not have the periodic points dense in the nonwandering set, but it seems likely that they can be modified so that this is the case. See [BF] and [FY].

Other questions of interest arise from our choices of candidates for the notions of "indecomposability" and "permanence" (chain recurrence and persistence, respectively). As I mentioned in the introduction, persistence is the weakest viable 
notion of permanence; the question is whether there is a stronger version that also retains genericity.

Concerning the "indecomposability" side of things, the main problem is to describe the internal structure of the chain transitive attractors and quasi-attractors as much as possible. One question that comes immediately to mind is whether these quasi-attractors are nonwandering. This is related to the question of whether $C R(f)$ is generically equal to $\Omega(f)$. This is a difficult question that has received attention in the past, in the work of Shub, Smale, and Nitecki on fine sequences of filtrations. $(C R(f)=\Omega(f)$ if and only if $f$ has a fine sequence of filtrations; see [SS], [NS], [Sh2], and [Sh3].)

Finally, I should discuss the applicability of the results of this paper to vector fields and diffeomorphisms as well as to flows. Flows were chosen over these alternatives as the central object of discussion because, first, continuous dynamical systems present some advantages in explication over discrete dynamical systems; and secondly, because the process of suspension naturally transforms $C^{r}$ diffeomorphisms into $C^{r}$ flows. (This was useful because most of the relevant examples described in the literature are discussed as diffeomorphisms rather than as flows.) However, neither of these considerations is vital, and it is apparent how to make the relevant changes so that all of the results contained herein apply as well to vector fields and diffeomorphisms. This kind of argument is contained in [Bo, $\S 10]$.

\section{BIBLIOGRAPHY}

[B1] R. Bowen, Equilibrium states and the ergodic theory of Anosov diffeomorphisms, Springer-Verlag, New York, 1975.

[BF] R. Bowen and J. Franks, The periodic points of maps of the disk and the interval, Topology 15 (1976), 337-342.

[Bo] H. G. Bothe, A modification of the Kupka-Smale theorem and smooth invariant manifolds of dynamical systems, Math. Nachr. 89 (1979), 25-42.

[BR] R. Bowen and D. Ruélle, The ergodic theory of Axiom A flows, Invent. Math. 29 (1975), 181-222.

[C] C. Conley, Isolated invariant sets and the Morse index, Amer. Math. Soc., Providence, R. I., 1978.

[FY] J. Franks and L. S. Young, preprint, Northwestern Univ., 1980.

[G] J. Guckenheimer, A strange, strange attractor, The Hopf Bifurcation and its Applications, (J. Marsden and M. McCracken, editors), Springer-Verlag, Berlin and New York, 1976.

[GA] S. Smale and S. S. Chern (Editors), Global analysis, Proc. Sympos. Pure Math., vol. 14, Amer. Math. Soc., Providence, R. I., 1970.

[H] P. Hartman, Ordinary differential equations, Hartman, Baltimore, Md., 1973.

[Hi] M. Hirsch, Differential topology, Springer-Verlag, New York, 1976.

[HPS] M. Hirsch, C. Pugh and M. Shub, Invariant manifolds, Springer-Verlag, New York, 1977.

[K] K. Kuratowski, Topology, Vol. I (J. Jaworowski, translator), Academic Press, New York, 1966.

[Lo] A. O. Lopes, Structural stability and hyperbolic attractors, Trans. Amer. Math. Soc. 252 (1979), 205-219.

[M] A. Manning (Editor), Dynamical systems-Warwick 1974, Lecture Notes in Math., vol. 468, Springer-Verlag, New York, 1975.

[N] Z. Nitecki, Differential dynamics, M.I.T. Press, Cambridge, Mass., 1971.

[NS] A. Nitecki and M. Shub, Filtrations, decompositions, and explosions, Amer. J. Math. 97 (1976), 1029-1047.

[Na] S. Nadler, Hyperspaces of sets, Dekker, New York, 1978.

[Ne1] S. Newhouse, Non-density of Axiom $A(a)$ on $S^{2}$, Global Analysis, Proc. Sympos. Pure Math., vol. 14, Amer. Math. Soc., Providence, R. I., 1970, pp. 191-203.

[Ne2] ___ Diffeomorphisms with infinitely many sinks, Topology 13 (1974), 9-18.

[O] M. M. C. de Oliveira, $C^{0}$-density of structurally stable vector fields, Bull. Amer. Math. Soc. 82 (1976), 786. 
[P] C. Pugh, An improved closing lemma and a general density theorem, Amer. J. Math. 89 (1967), 1010-1021.

[PP] J. Palis, C. Pugh, M. Shub and D. Sullivan, Genericity theorems in topological dynamics, Dynamical Systems, Lecture Notes in Math., vol. 468, Springer-Verlag, New York, 1975, pp. 241-250.

[S] S. Smale, Differentiable dynamical systems, Bull. Amer. Math. Soc. 73 (1967), 747-817.

[Sh1] M. Shub, Structurally stable diffeomorphisms are dense, Bull. Amer. Math. Soc. 78 (1972), 817-818.

[Sh2] Dynamical systems, filtrations and entropy, Bull. Amer. Math. Soc. 80 (1974), 27-41.

[Sh3] _ Stability and genericity for diffeomorphisms, Dynamical Systems (M. M. Peixoto, editor), Academic Press, New York, 1973, pp. 493-515.

[SS] M. Shub and S. Smale, Beyond hyperbolicity, Ann. of Math. (2) 96 (1972), 587-591.

[T] R. Thom, Structural stability and morphogenesis, (D. H. Fowler, translator), Benjamin, Reading, Mass., 1975.

[Ta1] F. Takens, On Zeeman's tolerance stability conjecture, Manifolds-Amsterdam, 1970, Lecture Notes in Math., vol. 197, Springer-Verlag, New York, 1971, pp. 209-219.

[Ta2] __ Tolerance stability, Dynamical Systems, Lecture Notes in Math., vol. 468, SpringerVerlag, New York, 1975, pp. 293-304.

[Wi1] R. F. Williams, The "D.A." maps of Smale and structural stability, Global Analysis, Proc. Sympos. Pure Math., vol. 14, Amer. Math. Soc., Providence, R. I., 1970, pp. 329-334.

[Wi2] The structure of Lorenz attractors, Turbulence Seminar, Lecture Notes in Math., vol. 615, Springer-Verlag, New York, 1977, pp. 94-112.

[Wi3] __ The structure of Lorenz attractors, preprint, Northwestern Univ., 1978.

[Z] E. C. Zeeman, Morse inequalities for diffeomorphisms with shoes and flows with solenoids, Dynamical Systems, Lecture Notes in Math., vol. 468, Springer-Verlag, New York, 1975, pp. 44-47.

Department of Mathematics, Case Western Reserve University, Cleveland, OHio 44106 